\title{
CR STATISTICAL SUBMANIFOLDS
}

\author{
Mirjana MILIJEVIĆ \\ (Received 4 November 2017 and revised 25 August 2018)
}

\begin{abstract}
The non-existence of CR submanifolds of maximal CR dimension with umbilical shape operator in holomorphic statistical manifolds is proven. Our results are a generalization of the known results in the theory of CR submanifolds in complex space forms. Statistical manifolds in this paper are considered as manifolds consisting of certain probability density functions. In this setting we have two shape operators in the distinguished normal vector field direction with respect to the affine connection of the ambient space, and the one with respect to the dual connection. After obtaining the fundamental equations for CR submanifolds in holomorphic statistical manifolds, we examine umbilical dual shape operators.
\end{abstract}

\section{Introduction}

CR submanifolds in Kähler geometry were introduced by A. Bejancu in 1978 as a generalization of both complex submanifolds and totally real submanifolds. The works of K. Yano and M. Kon [9] and A. Bejancu [1] gather the significant results on this topic. M. Djorić and M. Okumura have intensively investigated CR submanifolds in the complex projective space, with focus on the case when CR submanifolds are of maximal CR dimension. They generalized many important results on real hypersurfaces (Y. Tashiro and S. Tachibana [7], R. Takagi [6], among others), which are typical examples of CR submanifolds of maximal CR dimension.

Soon after CR submanifolds in Kähler manifolds were introduced, their research was extended to other ambient spaces. There is a large amount of literature when the ambient manifold is a nearly Kähler or locally conformal Kähler manifold. We initiate investigation of CR submanifolds in holomorphic statistical manifolds, which are new objects originating from information geometry. Statistical manifolds may be considered as manifolds consisting of certain probability density functions. They are geometrically formulated as Riemannian manifolds with a certain affine connection. Their complex version, i.e. holomorphic statistical manifolds, were defined by T. Kurose in 2004.

After obtaining the fundamental equations for CR submanifolds in holomorphic statistical manifolds, we investigate CR submanifolds with umbilical shape operators. We show that the results obtained by M. Djorić and M. Okumura [2] and by Y. Tashiro and S. Tachibana [7] hold in the theory of CR submanifolds in holomorphic statistical manifolds, by finding the corresponding conditions to the ones in the original theory.

When $M$ is a CR submanifold of maximal CR dimension in a holomorphic statistical manifold, we naturally get a special normal vector field, which is called the distinguished

2010 Mathematics Subject Classification: Primary 53C15, 53C40, 53B20.

Keywords: holomorphic statistical manifolds; CR submanifolds.

(C) 2019 Faculty of Mathematics, Kyushu University 
normal vector field of $M$. Accordingly, in this setting we have two shape operators $A$ and $A^{*}$, which are the shape operator in the distinguished normal vector field direction with respect to the affine connection of the ambient space, and the one with respect to the dual connection. We then obtain the following.

THEOREM 1. Let $M$ be an n-dimensional CR submanifold of maximal CR dimension in an $(n+p)$-dimensional holomorphic statistical manifold $\bar{M}$ of constant holomorphic sectional curvature $c$ and let $p<(n+1) / 2$. If for the shape operators $A$ and $A^{*}$ of the distinguished normal vector field $\xi$, and functions $\alpha$ and $\beta, A X=\alpha X$ and $A^{*} X=\beta X$, then $c=0$.

\section{Preliminaries}

At first we will give a definition of CR submanifolds of (maximal) CR dimension. They are defined by R. Nirenberg and R. O. Wells, Jr. [4] as submanifolds with holomorphic tangent space of constant dimension.

Let $(\bar{M}, \bar{g})$ be a complex manifold with the complex structure $J$ and let $(M, g)$ be a submanifold of $\bar{M}$ with tangent space $T_{x} M$. With $\bar{g}$ we denote a Hermitian metric on $\bar{M}$, and with $g$ the induced metric on $M$. We call $H_{x} M=J T_{x} M \cap T_{x} M, x \in M$, the holomorphic tangent space of $M$.

Definition 1. If $H_{x} M$ has constant dimension with respect to $x \in M$, the submanifold $M$ is called a Cauchy-Riemann submanifold or briefly CR submanifold and the constant complex dimension is called the CR dimension of $M$.

Let $M$ be a CR submanifold of real dimension $n$ in a manifold $\bar{M}$ of real dimension $n+p$. We say that $M$ is a CR submanifold of maximal CR dimension if the real dimension of $H_{x} M$ is $n-1, x \in M$. Therefore, $M$ is necessarily odd-dimensional and there exists a unit vector $\xi_{x}$ normal to $T_{x} M$ with respect to $\bar{g}$, such that

$$
J T_{x} M \subset T_{x} M \oplus \operatorname{span}\left\{\xi_{x}\right\}, \quad x \in M .
$$

Hence, for any $X \in \Gamma(T M)$, we may write

$$
J X=P X+u(X) \xi,
$$

where $P$ is an endomorphism acting on $T M$ and $u$ is a 1-form on $M$.

LEMMA 1. [2] The subbundle $T_{1}^{\perp} M=\left\{\eta \in T^{\perp} M \mid \bar{g}(\eta, \xi)=0\right\}$ is J-invariant and we can choose a local orthonormal basis of $T^{\perp} M$ in the following way:

$$
\xi, \quad \xi_{1}, \ldots, \xi_{q}, \quad \xi_{1^{*}}, \ldots, \xi_{q^{*}},
$$

where $\xi_{a^{*}}=J \xi_{a}, a=1, \ldots, q$ and $q=(p-1) / 2$.

Moreover, we have

$$
\begin{gathered}
J \xi=-U \in \Gamma(T M), \\
P^{2} X=-X+u(X) U, \\
u(P X)=0, \quad P U=0, \\
g(U, U)=1, \quad g(U, X)=u(X) .
\end{gathered}
$$


For more details on how to derive these formulas, we refer to [2].

Next, we give a definition of (holomorphic) statistical manifolds and their sectional curvature. We will work with sectional curvature defined by B. Opozda (see [5]), with a remark that this definition comes after the one given by Kurose (2004) which is used in statistical manifolds research as well.

Let $\bar{M}$ be a $C^{\infty}$ manifold of dimension $\bar{m} \geq 2, \bar{\nabla}$ an affine connection on $\bar{M}$, and $\bar{g}$ a Riemannian metric on $\bar{M}$.

\section{Definition 2.}

(1) $(\bar{M}, \bar{\nabla}, \bar{g})$ is called a statistical manifold if

(i) $\bar{\nabla}$ is torsion-free and

(ii) $\left(\bar{\nabla}_{X} \bar{g}\right)(Y, Z)=\left(\bar{\nabla}_{Y} \bar{g}\right)(X, Z)$ for $X, Y, Z \in \Gamma(T \bar{M})$.

(2) $\bar{\nabla}^{*}$ is called the dual connection of $\bar{\nabla}$ with respect to $\bar{g}$ if

$$
X \bar{g}(Y, Z)=\bar{g}\left(\bar{\nabla}_{X} Y, Z\right)+\bar{g}\left(Y, \bar{\nabla}_{X}^{*} Z\right), \quad X, Y, Z \in \Gamma(T \bar{M}) .
$$

Definition 3. [5] For a statistical manifold $(\bar{M}, \bar{\nabla}, \bar{g})$ we define

$$
\bar{S}^{(\bar{\nabla}, \bar{g})}(X, Y) Z=\frac{1}{2}\left\{\bar{R}^{\bar{\nabla}}(X, Y) Z+\bar{R}^{*}(X, Y) Z\right\},
$$

and

$$
\bar{S}(X, Y, Z, W)=\bar{g}\left(\bar{S}^{(\bar{\nabla}, \bar{g})}(Z, W) Y, X\right), \quad X, Y, Z, W \in \Gamma(T \bar{M}),
$$

where $\bar{R} \bar{\nabla}$ and $\bar{R}^{*}$ are the curvature tensors of $\bar{\nabla}$ and $\bar{\nabla}^{*}$, respectively.

Definition 4. [3] The sectional curvature of a statistical manifold $(\bar{M}, \bar{\nabla}, \bar{g})$ is constant $c$ if and only if

$$
\bar{S}^{(\bar{\nabla}, \bar{g})}(X, Y) Z=c\{\bar{g}(Y, Z) X-\bar{g}(X, Z) Y\}
$$

for $X, Y, Z \in \Gamma(T \bar{M})$.

To obtain the fundamental equations for CR submanifolds in holomorphic statistical manifolds, we use equations given by P. W. Vos [8] which are fundamental in statistical submanifolds theory.

Definition 5. Let $(\bar{M}, \bar{\nabla}, \bar{g})$ be a statistical manifold, and $M$ a submanifold of $\bar{M}$. By $T_{x}^{\perp} M$ we denote the normal space of $M$, i.e. $T_{x}^{\perp} M:=\left\{v \in T_{x} \bar{M} \mid \bar{g}(v, w)=0, w \in T_{x} M\right\}$, and by $g$ the induced metric on $M$. We define

$$
\begin{aligned}
& \nabla, \nabla^{*}: \Gamma(T M) \times \Gamma(T M) \rightarrow \Gamma(T M), \\
& h, h^{*}: \Gamma(T M) \times \Gamma(T M) \rightarrow \Gamma\left(T^{\perp} M\right), \\
& A, A^{*}: \Gamma\left(T^{\perp} M\right) \times \Gamma(T M) \rightarrow \Gamma(T M),
\end{aligned}
$$

and

$$
D, D^{*}: \Gamma(T M) \times \Gamma\left(T^{\perp} M\right) \rightarrow \Gamma\left(T^{\perp} M\right)
$$


by

$$
\begin{aligned}
& \bar{\nabla}_{X} Y=\nabla_{X} Y+h(X, Y), \quad \bar{\nabla}_{X} V=-A_{V} X+D_{X} V, \\
& \bar{\nabla}_{X}^{*} Y=\nabla_{X}^{*} Y+h^{*}(X, Y), \quad \bar{\nabla}_{X}^{*} V=-A_{V}^{*} X+D_{X}^{*} V,
\end{aligned}
$$

for $X, Y \in \Gamma(T M), V \in \Gamma\left(T^{\perp} M\right)$.

Proposition 1. Let $M, g, \nabla, \nabla^{*}, h, h^{*}, A, A^{*}, D$ and $D^{*}$ be as in Definition 5. Then we have the following.

(1) $(M, \nabla, g)$ and $\left(M, \nabla^{*}, g\right)$ are statistical manifolds, and $\nabla^{*}$ is the dual of $\nabla$ with respect to $g$.

(2) $D$ and $D^{*}$ are connections of $T^{\perp} M \rightarrow M$. Denoting by $g^{\perp}$ the metric of $T^{\perp} M$ induced from $\bar{g}$, we have

$$
X g^{\perp}(U, V)=g^{\perp}\left(D_{X} U, V\right)+g^{\perp}\left(U, D_{X}^{*} V\right), \quad U, V \in \Gamma\left(T^{\perp} M\right), X \in \Gamma(T M) .
$$

(3) $h, h^{*}, A$ and $A^{*}$ are tensor fields satisfying

$$
\begin{gathered}
h(X, Y)=h(Y, X), \quad h^{*}(X, Y)=h^{*}(Y, X), \\
g\left(A_{V} X, Y\right)=\bar{g}\left(h^{*}(X, Y), V\right), \quad g\left(A_{V}^{*} X, Y\right)=\bar{g}(h(X, Y), V), \\
X, Y \in \Gamma(T M), \quad V \in \Gamma\left(T^{\perp} M\right) .
\end{gathered}
$$

From the last proposition, we conclude that

$$
g\left(A_{V} X, Y\right)=g\left(X, A_{V} Y\right), \quad g\left(A_{V}^{*} X, Y\right)=g\left(X, A_{V}^{*} Y\right)
$$

for $X, Y \in \Gamma(T M), V \in \Gamma\left(T^{\perp} M\right)$. That is, $A_{V}$ and $A_{V}^{*}$ are symmetric with respect to $g$.

Definition 6. Let $(\bar{M}, J, \bar{g})$ be a Kähler manifold and $\bar{\nabla}$ an affine connection of $\bar{M}$. Then $(\bar{M}, \bar{\nabla}, \bar{g}, J)$ is called a holomorphic statistical manifold if

(1) $(\bar{M}, \bar{\nabla}, \bar{g})$ is a statistical manifold and

(2) $\bar{\omega}:=\bar{g}(*, J *)$ is a $\bar{\nabla}$-parallel 2-form on $\bar{M}$.

We know that, in Kähler manifolds, complex structure is parallel. This is not the case in holomorphic statistical manifolds. Instead, we have the following property.

LeMma 2. Let $(\bar{M}, \bar{\nabla}, \bar{g}, J)$ be a holomorphic statistical manifold. Then, $\bar{\nabla}_{X} J Y=J \bar{\nabla}_{X}^{*} Y$, for $X, Y \in \Gamma(T \bar{M})$, where $\bar{\nabla}^{*}$ is the dual connection of $\bar{\nabla}$ with respect to $\bar{g}$.

Definition 7. [3] A holomorphic statistical manifold $(\bar{M}, \bar{\nabla}, \bar{g}, J)$ is said to be of constant holomorphic sectional curvature $c \in \mathbb{R}$ if

$$
\begin{aligned}
\bar{S}^{(\bar{\nabla}, \bar{g})}(X, Y) Z= & \frac{c}{4}\{\bar{g}(Y, Z) X-\bar{g}(X, Z) Y \\
& +\bar{g}(J Y, Z) J X-\bar{g}(J X, Z) J Y+2 \bar{g}(X, J Y) J Z\}
\end{aligned}
$$

for $X, Y, Z \in \Gamma(T \bar{M})$. 


\section{Statistical hypersurfaces}

This section is dedicated to typical examples of CR statistical submanifolds (of maximal $\mathrm{CR}$ dimension), i.e. statistical hypersurfaces. We will generalize here the famous result of Tashiro and Tachibana from 1963, about umbilical hypersurfaces. This will be expressed in our Theorem 2.

To that purpose, we assume that $(\bar{M}, \bar{\nabla}, \bar{g}, J)$ is a $2 m$-dimensional holomorphic statistical manifold and that $M$ is a $(2 m-1)$-dimensional submanifold of $\bar{M}$, i.e. a hypersurface of $\bar{M}$. The Gauss and Weingarten equations are given by:

$$
\begin{array}{ll}
\bar{\nabla}_{X} Y=\nabla_{X} Y+h(X, Y), & \bar{\nabla}_{X}^{*} Y=\nabla_{X}^{*} Y+h^{*}(X, Y), \\
\bar{\nabla}_{X} \xi=-A X+s(X) \xi, & \bar{\nabla}_{X}^{*} \xi=-A^{*} X+s^{*}(X) \xi,
\end{array}
$$

respectively. For the coefficients $s$ and $s^{*}, s(X)=-s^{*}(X)$ holds.

The structure vector $U$ is defined by

$$
U:=-J \xi \in \Gamma(T M) .
$$

For $X \in \Gamma(T M), J X$ decomposes to the tangent and the normal parts as:

$$
J X=P X+g(X, U) \xi .
$$

When $\bar{M}$ is of constant holomorphic sectional curvature $c$, the Codazzi equation is of the following form:

$$
\begin{gathered}
\frac{c}{2}\{g(X, U) P Y-g(Y, U) P X+2 g(X, P Y) U\} \\
=\left(\nabla_{X}^{*} A^{*}+\nabla_{X} A\right) Y-\left(\nabla_{Y}^{*} A^{*}+\nabla_{Y} A\right) X \\
\quad+s(X)\left(A^{*}-A\right) Y-s(Y)\left(A^{*}-A\right) X .
\end{gathered}
$$

Using equation (9), we will prove our main result of this section contained in Theorem 2, motivated by Example 1 below.

Example 1. Let $(\bar{M}, \bar{\nabla}, \bar{g})$ be the $(n+1)$-dimensional upper half Hessian space of constant Hessian curvature 4 which is constructed as follows.

$(\bar{M}, \bar{g})$ denotes the upper half space of constant curvature -1 , i.e.

$$
\begin{gathered}
\bar{M}:=\left\{x=\left(x^{1}, \ldots, x^{n+1}\right)^{t} \in \mathbb{R}^{n+1} \mid x^{n+1}>0\right\}, \\
\bar{g}:=\left(x^{n+1}\right)^{-2} \sum_{i=1}^{q} d x^{i} d x^{i} .
\end{gathered}
$$

On $\bar{M}$, we define an affine connection $\bar{\nabla}$ by

$$
\begin{gathered}
\bar{\nabla}_{\partial / \partial x^{n+1}} \frac{\partial}{\partial x^{n+1}}=\left(x^{n+1}\right)^{-1} \frac{\partial}{\partial x^{n+1}}, \quad \bar{\nabla}_{\partial / \partial x^{i}} \frac{\partial}{\partial x^{j}}=2 \delta_{i j}\left(x^{n+1}\right)^{-1} \frac{\partial}{\partial x^{n+1}}, \\
\bar{\nabla}_{\partial / \partial x^{i}} \frac{\partial}{\partial x^{n+1}}=\bar{\nabla}_{\partial / \partial x^{n+1}} \frac{\partial}{\partial x^{i}}=0, \quad i, j=1, \ldots, n .
\end{gathered}
$$

For a constant $x_{0}>0$, we define an immersion $f$ by

$$
\left(x^{1}, \ldots, x^{n}\right) \mapsto\left(x^{1}, \ldots, x^{n}, x^{0}\right) \in \bar{M} .
$$


We can easily calculate the shape operators of the normal vector field $\xi=x_{0} \partial / \partial x^{n+1}, A^{*}=0$ and $A=2 I$.

THEOREM 2. Let $M$ be a real hypersurface of a holomorphic statistical manifold $\bar{M}$ of constant holomorphic sectional curvature c. If for the shape operators $A$ and $A^{*}$ of $M$, and functions $\alpha$ and $\beta, A X=\alpha X$ and $A^{*} X=\beta X$ for $X \in \Gamma(T M)$, then $c=0$.

Proof. Differentiating $A X=\alpha X$, we get

$$
\left(\nabla_{Y} A\right) X+A \nabla_{Y} X=(Y \alpha) X+\alpha \nabla_{Y} X,
$$

i.e.

$$
\left(\nabla_{Y} A\right) X=(Y \alpha) X
$$

Similarly, we get that

$$
\left(\nabla_{Y}^{*} A^{*}\right) X=(Y \beta) X .
$$

From the Codazzi equation (9), (10) and (11), we have

$$
\begin{gathered}
X(\alpha+\beta) Y-Y(\alpha+\beta) X+(\beta-\alpha)(s(X) Y-s(Y) X) \\
\quad=\frac{c}{2}\{g(X, U) P Y-g(Y, U) P X+2 g(X, P Y) U\} .
\end{gathered}
$$

Now, we put $Y=U$ in equation (12). We get

$$
X(\alpha+\beta) U-U(\alpha+\beta) X+(\beta-\alpha)(s(X) U-s(U) X)=-\frac{c}{2} P X .
$$

From this equation, we get

$$
\begin{aligned}
& X(\alpha+\beta) g(U, P X)-U(\alpha+\beta) g(X, P X)+(\beta-\alpha)(s(X) g(U, P X)-s(U) g(X, P X)) \\
& \quad=-\frac{c}{2} g(P X, P X),
\end{aligned}
$$

that is, $c=0$.

We note that this theorem follows directly from Theorem 1 but we gave its proof independently.

Remark 1. A corollary of Theorem 2 in the case when the ambient manifold is Kählerian is Tashiro and Tachibana's result from 1963 about the non-existence of umbilical hypersurfaces in non-flat complex space forms.

PROPOSITION 2. Let $M$ be a real hypersurface of a holomorphic statistical manifold $\bar{M}$ of constant holomorphic sectional curvature c. If for the shape operators $A$ and $A^{*}$ of $M$, and functions $\alpha$ and $\beta, \alpha \neq \beta, A X=\alpha X$, and $A^{*} X=\beta X$ for $X \in \Gamma(T M)$, then $\alpha+\beta=$ const. if and only if $\xi$ is parallel with respect to the normal connection $D\left(D^{*}\right)$.

Proof. From Theorem 2, using the Codazzi equation (12), we get

$$
X(\alpha+\beta) U-U(\alpha+\beta) X+(\beta-\alpha)(s(X) U-s(U) X)=0 .
$$


Next, we apply the operator $P$ to the both sides of the last equation. The result is

$$
U(\alpha+\beta) P X+(\beta-\alpha) s(U) P X=0 .
$$

From the last equation it follows that

$$
U(\alpha+\beta)=(\alpha-\beta) s(U) .
$$

Now, from the last equation and (13), we get

$$
X(\alpha+\beta)=(\alpha-\beta) s(X) .
$$

Since $\alpha \neq \beta$, the conclusion follows.

\section{CR statistical submanifolds of maximal CR dimension}

After obtaining the non-existence results on statistical hypersurfaces it is natural to ask whether they are valid in their generalization, CR statistical submanifolds of maximal CR dimension. In this section we will impose the same conditions on the shape operators of the distinguished normal to a CR statistical submanifold of maximal CR dimension, as in the case of the normal vector of a statistical hypersurface. The result is our Theorem 1, given in the Introduction. Here we show its proof following the fundamental equations that are derived on CR statistical submanifolds of maximal CR dimension.

With $M$ we denote an $n$-dimensional $\mathrm{CR}$ submanifold of maximal CR dimension in a holomorphic statistical manifold $\bar{M}$ of dimension $n+p, p>1$. Let $\xi, \xi_{1}, \ldots, \xi_{q}$, $\xi_{1^{*}}, \ldots, \xi_{q^{*}}, q=(p-1) / 2$, be orthonormal frame fields of $T^{\perp} M$, as in Lemma 1 . We define their shape operators with respect to $\bar{\nabla}$ as

$$
A:=A_{\xi}, \quad A_{1}:=A_{\xi_{1}}, \quad \ldots, \quad A_{q}:=A_{\xi_{q}}, \quad A_{1^{*}}:=A_{\xi_{1^{*}}}, \quad \ldots, \quad A_{q^{*}}:=A_{\xi_{q^{*}}},
$$

and the shape operators with respect to $\bar{\nabla}^{*}$ as

$$
A^{*}:=A_{\xi}^{*}, \quad A_{1}^{*}:=A_{\xi_{1}}^{*}, \quad \ldots, \quad A_{q}^{*}:=A_{\xi_{q}}^{*}, \quad A_{1^{*}}^{*}:=A_{\xi_{1^{*}}}^{*}, \quad \ldots, \quad A_{q^{*}}^{*}:=A_{\xi_{q^{*}}}^{*} .
$$

We can write the normal connections $D_{X} \xi$ and $D_{X}^{*} \xi$ as

$$
\begin{aligned}
& D_{X} \xi=s(X) \xi+\sum_{a=1}^{q}\left\{s_{a}(X) \xi_{a}+s_{a^{*}}(X) \xi_{a^{*}}\right\} \\
& D_{X}^{*} \xi=s^{*}(X) \xi+\sum_{a=1}^{q}\left\{s_{a}^{*}(X) \xi_{a}+s_{a^{*}}^{*}(X) \xi_{a^{*}}\right\} .
\end{aligned}
$$

From (14) and $\left(14^{*}\right)$, we have the following Weingarten equations:

$$
\begin{aligned}
& \bar{\nabla}_{X} \xi=-A X+D_{X} \xi=-A X+s(X) \xi+\sum_{a=1}^{q}\left\{s_{a}(X) \xi_{a}+s_{a^{*}}(X) \xi_{a^{*}}\right\}, \\
& \bar{\nabla}_{X}^{*} \xi=-A^{*} X+D_{X}^{*} \xi=-A^{*} X+s^{*}(X) \xi+\sum_{a=1}^{q}\left\{s_{a}^{*}(X) \xi_{a}+s_{a^{*}}^{*}(X) \xi_{a^{*}}\right\},
\end{aligned}
$$




$$
\begin{aligned}
& \bar{\nabla}_{X} \xi_{a}=-A_{a} X+D_{X} \xi_{a}=-A_{a} X-s_{a}^{*}(X) \xi+\sum_{b=1}^{q}\left\{s_{a b}(X) \xi_{b}+s_{a b^{*}}(X) \xi_{b^{*}}\right\}, \\
& \bar{\nabla}_{X}^{*} \xi_{a}=-A_{a}^{*} X+D_{X}^{*} \xi_{a}=-A_{a}^{*} X-s_{a}(X) \xi+\sum_{b=1}^{q}\left\{s_{a b}^{*}(X) \xi_{b}+s_{a b^{*}}^{*}(X) \xi_{b^{*}}\right\}, \\
& \bar{\nabla}_{X} \xi_{a^{*}}=-A_{a^{*}} X+D_{X} \xi_{a^{*}}=-A_{a^{*}} X-s_{a^{*}}^{*}(X) \xi+\sum_{b=1}^{q}\left\{s_{a^{*} b}(X) \xi_{b}+s_{a^{*} b^{*}}(X) \xi_{b^{*}}\right\}, \\
& \bar{\nabla}_{X}^{*} \xi_{a^{*}}=-A_{a^{*}}^{*} X+D_{X}^{*} \xi_{a^{*}}=-A_{a^{*}}^{*} X-s_{a^{*}}(X) \xi+\sum_{b=1}^{q}\left\{s_{a^{*} b}^{*}(X) \xi_{b}+s_{a^{*} b^{*}}^{*}(X) \xi_{b^{*}}\right\} .
\end{aligned}
$$

Now, we will differentiate the equation $\xi_{a^{*}}=J \xi_{a}$, and use the obtained equations (15), (16) and (17), with their dual equations. The results are the following five equations:

$$
\begin{gathered}
A_{a^{*}} X=P A_{a}^{*} X-s_{a}(X) U, \quad A_{a^{*}}^{*}=P A_{a} X-s_{a}^{*}(X) U, \\
A_{a} X=-P A_{a^{*}}^{*} X+s_{a^{*}}(X) U, \quad A_{a}^{*} X=-P A_{a^{*}} X+s_{a^{*}}^{*}(X) U, \\
s_{a^{*}}^{*}(X)=u\left(A_{a}^{*} X\right)=g\left(A_{a}^{*} X, U\right), \quad s_{a^{*}}(X)=u\left(A_{a} X\right)=g\left(A_{a} X, U\right), \\
s_{a}^{*}(X)=-u\left(A_{a^{*}}^{*} X\right)=-g\left(A_{a^{*}}^{*} X, U\right), \quad s_{a}(X)=-u\left(A_{a^{*}} X\right)=-g\left(A_{a^{*}} X, U\right), \\
s_{a^{*} b}=-s_{a b^{*}}^{*}, \quad s_{a^{*} b^{*}}=s_{a b}^{*}, \quad s_{a^{*} b}^{*}=-s_{a b^{*}}, \quad s_{a^{*} b^{*}}^{*}=s_{a b} .
\end{gathered}
$$

Further on, we will assume that our ambient manifold $\bar{M}$ is of constant holomorphic curvature $c$. Then, the Codazzi equation for the distinguished normal vector field $\xi \in$ $\Gamma\left(T^{\perp} M\right)$ is

$$
\begin{aligned}
\left(\nabla_{X}^{*} A^{*}+\nabla_{X} A\right) Y-\left(\nabla_{Y}^{*} A^{*}+\nabla_{Y} A\right) X \\
=\frac{c}{2}\{u(X) P Y-u(Y) P X-2 g(P X, Y) U\}+s(X)\left(A-A^{*}\right) Y-s(Y)\left(A-A^{*}\right) X \\
\quad+\sum_{a=1}^{q}\left\{s_{a}(X) A_{a} Y+s_{a^{*}}(X) A_{a^{*}} Y\right\}+\sum_{a=1}^{q}\left\{s_{a}^{*}(X) A_{a}^{*} Y+s_{a^{*}}^{*}(X) A_{a^{*}}^{*} Y\right\} \\
\quad-\sum_{a=1}^{q}\left\{s_{a}(Y) A_{a} X+s_{a^{*}}(Y) A_{a^{*}} X\right\}-\sum_{a=1}^{q}\left\{s_{a}^{*}(Y) A_{a}^{*} X+s_{a^{*}}^{*}(Y) A_{a^{*}}^{*} X\right\} .
\end{aligned}
$$

Proof of Theorem 1. Differentiating $A X=\alpha X, A^{*} X=\beta X$, and using the Codazzi equation (23), we get

$$
\begin{aligned}
& X(\alpha+\beta) Y-Y(\alpha+\beta) X \\
&=\frac{c}{2}\{u(X) P Y-u(Y) P X-2 g(P X, Y) U\}+s(X)(\alpha-\beta) Y-s(Y)(\alpha-\beta) X \\
&+\sum_{a=1}^{q}\left\{s_{a}(X) A_{a} Y+s_{a^{*}}(X) A_{a^{*}} Y\right\}+\sum_{a=1}^{q}\left\{s_{a}^{*}(X) A_{a}^{*} Y+s_{a^{*}}^{*}(X) A_{a^{*}}^{*} Y\right\} \\
&-\sum_{a=1}^{q}\left\{s_{a}(Y) A_{a} X+s_{a^{*}}(Y) A_{a^{*}} X\right\}-\sum_{a=1}^{q}\left\{s_{a}^{*}(Y) A_{a}^{*} X+s_{a^{*}}^{*}(Y) A_{a^{*}}^{*} X\right\} .
\end{aligned}
$$


If we take an inner product of (24) with the structure vector field, $U$, we get

$$
\begin{aligned}
X(\alpha+\beta) g(Y, U)-Y(\alpha+\beta) g(X, U) \\
=-c g(P X, Y)+s(X)(\alpha-\beta) u(Y)-s(Y)(\alpha-\beta) u(X) \\
+\sum_{a=1}^{q}\left\{s_{a}(X) g\left(A_{a} Y, U\right)+s_{a^{*}}(X) g\left(A_{a^{*}} Y, U\right)\right\} \\
\quad+\sum_{a=1}^{q}\left\{s_{a}^{*}(X) g\left(A_{a}^{*} Y, U\right)+s_{a^{*}}^{*}(X) g\left(A_{a^{*}}^{*} Y, U\right)\right\} \\
\quad-\sum_{a=1}^{q}\left\{s_{a}(Y) g\left(A_{a} X, U\right)+s_{a^{*}}(Y) g\left(A_{a^{*}} X, U\right)\right\} \\
\quad-\sum_{a=1}^{q}\left\{s_{a}^{*}(Y) g\left(A_{a}^{*} X, U\right)+s_{a^{*}}^{*}(Y) g\left(A_{a^{*}}^{*} X, U\right)\right\} .
\end{aligned}
$$

If we put $Y=U$ in (25), we get

$$
\begin{aligned}
X(\alpha+\beta)-U(\alpha+\beta) g(X, U) & \\
= & s(X)(\alpha-\beta)-s(U)(\alpha-\beta) u(X) \\
& +\sum_{a=1}^{q}\left\{s_{a}(X) s_{a^{*}}(U)-s_{a^{*}}(X) s_{a}(U)\right\}+\sum_{a=1}^{q}\left\{s_{a}^{*}(X) s_{a^{*}}^{*}(U)-s_{a^{*}}^{*}(X) s_{a}^{*}(U)\right\} \\
& -\sum_{a=1}^{q}\left\{s_{a}(U) s_{a^{*}}(X)-s_{a^{*}}(U) s_{a}(X)\right\}-\sum_{a=1}^{q}\left\{s_{a}^{*}(U) s_{a^{*}}^{*}(X)-s_{a^{*}}^{*}(U) s_{a}^{*}(X)\right\} .
\end{aligned}
$$

Next, we put $Y=U$ in (24), we obtain

$$
\begin{aligned}
& X(\alpha+\beta) U-U(\alpha+\beta) X \\
&=-\frac{c}{2} P X+s(X)(\alpha-\beta) U-s(U)(\alpha-\beta) X \\
&+\sum_{a=1}^{q}\left\{s_{a}(X) A_{a} U+s_{a^{*}}(X) A_{a^{*}} U\right\}+\sum_{a=1}^{q}\left\{s_{a}^{*}(X) A_{a}^{*} U+s_{a^{*}}^{*}(X) A_{a^{*}}^{*} U\right\} \\
&-\sum_{a=1}^{q}\left\{s_{a}(U) A_{a} X+s_{a^{*}}(U) A_{a^{*}} X\right\}-\sum_{a=1}^{q}\left\{s_{a}^{*}(U) A_{a}^{*} X+s_{a^{*}}^{*}(U) A_{a^{*}}^{*} X\right\} .
\end{aligned}
$$

From (26) and (27), we get

$$
\begin{aligned}
-U(\alpha+\beta) X+U(\alpha+\beta) u(X) U \\
=-\frac{c}{2} P X-s(U)(\alpha-\beta) X+s(U)(\alpha-\beta) u(X) U \\
\quad+\sum_{a=1}^{q}\left\{s_{a}(X) A_{a} U+s_{a^{*}}(X) A_{a^{*}} U\right\}+\sum_{a=1}^{q}\left\{s_{a}^{*}(X) A_{a}^{*} U+s_{a^{*}}^{*}(X) A_{a^{*}}^{*} U\right\}
\end{aligned}
$$




$$
\begin{aligned}
& -\sum_{a=1}^{q}\left\{s_{a}(U) A_{a} X+s_{a^{*}}(U) A_{a^{*}} X\right\}-\sum_{a=1}^{q}\left\{s_{a}^{*}(U) A_{a}^{*} X+s_{a^{*}}^{*}(U) A_{a^{*}}^{*} X\right\} \\
& -\sum_{a=1}^{q}\left\{s_{a}(X) s_{a^{*}}(U) U-s_{a^{*}}(X) s_{a}(U) U\right\}-\sum_{a=1}^{q}\left\{s_{a}^{*}(X) s_{a^{*}}^{*}(U) U-s_{a^{*}}^{*}(X) s_{a}^{*}(U) U\right\} \\
& +\sum_{a=1}^{q}\left\{s_{a}(U) s_{a^{*}}(X) U-s_{a^{*}}(U) s_{a}(X) U\right\}+\sum_{a=1}^{q}\left\{s_{a}^{*}(U) s_{a^{*}}^{*}(X) U-s_{a^{*}}^{*}(U) s_{a}^{*}(U) U\right\} .
\end{aligned}
$$

Now, we apply $P$ to (28). We get

$$
\begin{aligned}
-U(\alpha & +\beta) P X \\
= & -\frac{c}{2} P^{2} X-s(U)(\alpha-\beta) P X \\
& +\sum_{a=1}^{q}\left\{-s_{a}(X) P^{2} A_{a^{*}}^{*} U+s_{a^{*}}(X) P^{2} A_{a}^{*} U\right\}+\sum_{a=1}^{q}\left\{-s_{a}^{*}(X) P^{2} A_{a^{*}} U+s_{a^{*}}^{*}(X) P^{2} A_{a} U\right\} \\
& -\sum_{a=1}^{q}\left\{-s_{a}(U) P^{2} A_{a^{*}}^{*} X+s_{a^{*}}(U) P^{2} A_{a}^{*} X\right\}-\sum_{a=1}^{q}\left\{-s_{a}^{*}(U) P^{2} A_{a^{*}} X+s_{a^{*}}^{*}(U) P^{2} A_{a} X\right\},
\end{aligned}
$$

where we used (18) and (19). From (20), (21), (29) and (4), we get

$$
\begin{aligned}
-U & (\alpha+\beta) P X \\
= & -\frac{c}{2} P^{2} X-s(U)(\alpha-\beta) P X \\
& +\sum_{a=1}^{q}\left\{s_{a}(X) A_{a^{*}}^{*} U-s_{a^{*}}(X) A_{a}^{*} U\right\}+\sum_{a=1}^{q}\left\{s_{a}^{*}(X) A_{a^{*}} U-s_{a^{*}}^{*}(X) A_{a} U\right\} \\
& -\sum_{a=1}^{q}\left\{s_{a}(U) A_{a^{*}}^{*} X-s_{a^{*}}(U) A_{a}^{*} X\right\}-\sum_{a=1}^{q}\left\{s_{a}^{*}(U) A_{a^{*}} X-s_{a^{*}}^{*}(U) A_{a} X\right\} .
\end{aligned}
$$

We take an inner product of (30) by a vector field $Y$. When we interchange $X$ and $Y$ in the obtained equation, and then subtract the two obtained equations, we get

$$
U(\alpha+\beta)=s(U)(\alpha-\beta) .
$$

From the equations (31) and (26), we obtain

$$
\begin{aligned}
X(\alpha+\beta)= & s(X)(\alpha-\beta)+\sum_{a=1}^{q}\left\{2 s_{a}(X) s_{a^{*}}(U)-2 s_{a^{*}}(X) s_{a}(U)\right\} \\
& +\sum_{a=1}^{q}\left\{2 s_{a}^{*}(X) s_{a^{*}}^{*}(U)-2 s_{a^{*}}^{*}(X) s_{a}^{*}(U)\right\} .
\end{aligned}
$$


From (25) and (32), we conclude that

$$
\begin{aligned}
\sum_{a=1}^{q}\{ & \left.2 s_{a}(X) s_{a^{*}}(U) g(Y, U)-2 s_{a^{*}}(X) s_{a}(U) g(Y, U)\right\} \\
& +\sum_{a=1}^{q}\left\{2 s_{a}^{*}(X) s_{a^{*}}^{*}(U) g(Y, U)-2 s_{a^{*}}^{*}(X) s_{a}^{*}(U) g(Y, U)\right\} \\
& -\sum_{a=1}^{q}\left\{2 s_{a}(Y) s_{a^{*}}(U) g(X, U)-2 s_{a^{*}}(Y) s_{a}(U) g(X, U)\right\} \\
& -\sum_{a=1}^{q}\left\{2 s_{a}^{*}(Y) s_{a^{*}}^{*}(U) g(X, U)-2 s_{a^{*}}^{*}(Y) s_{a}^{*}(U) g(X, U)\right\} \\
= & -c g(P X, Y) \\
& +\sum_{a=1}^{q}\left\{s_{a}(X) g\left(A_{a} Y, U\right)+s_{a^{*}}(X) g\left(A_{a^{*}} Y, U\right)\right\} \\
& +\sum_{a=1}^{q}\left\{s_{a}^{*}(X) g\left(A_{a}^{*} Y, U\right)+s_{a^{*}}^{*}(X) g\left(A_{a^{*}}^{*} Y, U\right)\right\} \\
& -\sum_{a=1}^{q}\left\{s_{a}(Y) g\left(A_{a} X, U\right)+s_{a^{*}}(Y) g\left(A_{a^{*}} X, U\right)\right\} \\
& -\sum_{a=1}^{q}\left\{s_{a}^{*}(Y) g\left(A_{a}^{*} X, U\right)+s_{a^{*}}^{*}(Y) g\left(A_{a^{*}}^{*} X, U\right)\right\} .
\end{aligned}
$$

We change $X$ by $P X$ in (33). The resulting equation is

$$
\begin{aligned}
\sum_{a=1}^{q}\{ & \left.2 s_{a}(P X) s_{a^{*}}(U) U-2 s_{a^{*}}(P X) s_{a}(U) U\right\} \\
& +\sum_{a=1}^{q}\left\{2 s_{a}^{*}(P X) s_{a^{*}}^{*}(U) U-2 s_{a^{*}}^{*}(P X) s_{a}^{*}(U) U\right\} \\
= & -c P^{2} X \\
& +\sum_{a=1}^{q}\left\{s_{a}(P X) A_{a} U+s_{a^{*}}(P X) A_{a^{*}} U\right\}+\sum_{a=1}^{q}\left\{s_{a}^{*}(P X) A_{a}^{*} U+s_{a^{*}}^{*}(P X) A_{a^{*}}^{*} U\right\} \\
& \quad-\sum_{a=1}^{q}\left\{-s_{a^{*}}(P X) A_{a^{*}} U-s_{a}(P X) A_{a} U\right\}-\sum_{a=1}^{q}\left\{-s_{a^{*}}^{*}(P X) A_{a^{*}}^{*} U-s_{a}^{*}(P X) A_{a}^{*} U\right\},
\end{aligned}
$$

where we have used (20) and (21). 
In the last step we apply the operator $P$ to the last equation. We have the following:

$$
\begin{aligned}
c P^{3} X= & \sum_{a=1}^{q}\left\{2 s_{a}(P X) P A_{a} U+2 s_{a^{*}}(P X) P A_{a^{*}} U\right\} \\
& +\sum_{a=1}^{q}\left\{2 s_{a}^{*}(P X) P A_{a}^{*} U+2 s_{a^{*}}^{*}(P X) P A_{a^{*}}^{*} U\right\} .
\end{aligned}
$$

Since every tangent vector $Y$ orthogonal to $U$ can be expressed as $Y=P X$, for $n-1>4 q=$ $2(p-1)$ because of $p<(n+1) / 2$, it follows that there exists a unit vector field $Y=P X$ orthogonal to $\operatorname{span}\left\{A_{a} U, A_{a^{*}} U, A_{a}^{*} U, A_{a^{*}}^{*} U\right\}, a=1, \ldots, q$. For such $Y=P X$ it follows that $s_{a}(P X)=s_{a^{*}}(P X)=s_{a}^{*}(P X)=s_{a^{*}}^{*}(P X)=0, a=1, \ldots, q$, where we have used (20), (21) and (7). Now, from (35) it follows that $c P^{3} X=0$, that is $c=0$.

Following the proof above, we easily conclude the next proposition.

PROPOSITION 3. Let $M$ be a CR submanifold of maximal CR dimension in a holomorphic statistical manifold $\bar{M}$ of constant holomorphic sectional curvature $c$. If for the shape operators $A$ and $A^{*}$ of the distinguished normal vector field $\xi$, and functions $\alpha$ and $\beta$, $A X=\alpha X$ and $A^{*} X=\beta X$ for $X \in \Gamma(T M)$, then $c=0$ if there exists a tangent vector $X$ on $M$ satisfying that $X \neq 0, u(X)=0$, and $s_{a}(X)=s_{a^{*}}(X)=s_{a}^{*}(X)=s_{a^{*}}^{*}(X)=0, a=1, \ldots, q$.

COROLlary 1. Let $M$ be a CR submanifold of maximal CR dimension in a holomorphic statistical manifold $\bar{M}$ of constant holomorphic sectional curvature $c$. And let for the shape operators $A$ and $A^{*}$ of the distinguished normal vector field $\xi$, and functions $\alpha$ and $\beta$, $A X=\alpha X$ and $A^{*} X=\beta X$ for $X \in \Gamma(T M)$. If on $U^{\perp}=\{X \in T M \mid u(X)=0\}$ the vector field $\xi$ is parallel with respect to both $D$ and $D *$, then $c=0$.

We remark that this corollary generalizes Theorem 19.1 in [2].

In the case when the ambient manifold is Kählerian, we get the following.

COROLlary 2. Let $M$ be an n-dimensional $C R$ submanifold of maximal $C R$ dimension in an $(n+p)$-dimensional complex space form $\bar{M}$ of holomorphic sectional curvature $c$, and let $p<n$. If for the shape operator A of the distinguished normal vector field $\xi$, and a function $\alpha, A X=\alpha X$ for $X \in \Gamma(T M)$, then $c=0$.

Acknowledgements. The author thanks Professor H. Furuhata and Professor I. Hasegawa for their sincere help. Also, thanks to the referee's suggestions, this is an improved version of the paper.

\section{REFERENCES}

[1] A. Bejancu. Geometry of CR Submanifolds (Mathematics and its Applications). Springer, Berlin, 1986.

[2] M. Djorić and M. Okumura. CR Submanifolds of Complex Projective Space (Developments in Mathematics, 19). Springer, Berlin, 2009.

[3] H. Furuhata. Hypersurfaces in statistical manifolds. Differential Geom. Appl. 27 (2009), 420-429.

[4] R. Nirenberg and R. O. Wells, Jr. Approximation theorems on differentiable submanifolds of a complex manifold. Trans. Amer. Math. Soc. 142 (1965), 15-35.

[5] B. Opozda. Bochner's technique for statistical structures. Ann. Global. Anal. Geom. (2015), 357-395. 
[6] R. Takagi. On homogeneous real hypersurfaces in a complex projective space. Osaka J. Math. 10 (1973), 495-506.

[7] Y. Tashiro and S. Tachibana. On Fubinian and C-Fubinian manifolds. Kodai Math. Sem. Rep. 15 (1963), 176-183.

[8] P. W. Vos. Fundamental equations for statistical submanifolds with applications to the Bartlett correction. Ann. Inst. Statist. Math. 41(3) (1989), 429-450.

[9] K. Yano and M. Kon. CR Submanifolds of Kählerian and Sasakian Manifolds. Birkhäuser, Basel, 1983.

\author{
Mirjana Milijević \\ Department of Mathematics \\ Hokkaido University \\ North 10, West 8, Kita-ku \\ 060-0810 Sapporo \\ Japan \\ (E-mail: mirjana.mmilijevic@gmail.com)
}

